\title{
Research on Services Composition Optimization of Rectangular Cutting in Cloud Manufacturing
}

\author{
Zhaoyun $\mathrm{Wu}^{1, \mathrm{a}, *}$, Han Ling ${ }^{1, \mathrm{~b}}, \mathrm{Li} \mathrm{Li}^{2, \mathrm{c}}$, Lihui $\mathrm{Wu}^{1, \mathrm{~d}}$ and Nanbo Liu ${ }^{1, \mathrm{e}}$ \\ ${ }^{1}$ School of Mechanical \& Electrical Engineering, Henan University of Technology, Lianhua Street, \\ Zhengzhou, China \\ ${ }^{2}$ Automobile Engineering Department, Henan Polytechnic, Pingan Street, Zhengzhou, China \\ a'wzhaoyun@163.com, b1981524663@qq.com, ${ }^{\text {c }}$ cflw2006@163.com, ${ }^{d}$ wulihui@haut.edu.cn, \\ e $1 n b @$ haut.edu.cn \\ *corresponding author
}

Keywords: Services Composition Optimization, Rectangular Cutting, Cloud Manufacturing

\begin{abstract}
Based on research and analysis of the cloud services composition optimization problem, the cloud services composition optimization method of in cloud manufacturing was proposed in this paper. In order to effectively describe the cloud manufacturing services of rectangular cutting, the description model of cloud services was built. The model mainly included four elements: basic properties, processing capacity, real time state, and service quality attributes. According to the contents and requirements of cloud services modeling, the implementation framework based on three layers structure model was presented. Based on these, the cloud services composition flow of rectangular cutting was given, including decomposing task, selecting cloud services, and combining cloud services. The method of this paper plays an important role in research on the optimization algorithm of cloud services composition.
\end{abstract}

\section{Introduction}

Cloud manufacturing is a new concept of networked remote manufacturing based on service concept. It overcomes the obstacle of geographical location and can integrate many manufacturing resources distributed in different regions through the cloud service platform, and then provide services such as cloud service discovery, matching, combination and invocation for users in different regions[1-2]. For the rectangular cutting cloud manufacturing service platform, according to the user's actual needs and the release of the cutting task to quickly generate a viable cloud service portfolio, and the overall optimization of cloud service composition scheme are very important for the efficient utilization of rectangular cutting clouds and manufacturing resources. Therefore, it is of great theoretical and practical value to study the cloud services composition optimization method for rectangular cutting.

\section{Description Model of Rectangular Cutting Cloud Service}

In order to effectively describe the cloud manufacturing service for rectangular cutting, this paper constructs the description model from four aspects: basic properties, processing capacity, real time state, and service quality attributes, as shown in Figure 1.

According to the description model of the rectangle cut cloud service, the following formal description and definition are made by the set method.

Definition 1: Rectangular cutting cloud services, Rectangular cutting cloud services RCCS can be represented in a quaternion form, RCCS (Rectangular Cutting Cloud Service $)=\{$ RCCSBasicInfo, RCCSCapaInfo, RCCSStatusInfo, RCCSQoS\}.

Definition 2: The basic properties of the rectangular cutting cloud services, $\mathrm{RCCSBasicInfo}=$ \{RCCSID, RCCSName, RCCSSManufacturer, RCCSAddress $\}$.

Definition 3: Processing capability attributes of the rectangular cutting cloud services, 
RCCSCapaInfo $=$ \{RCCSType, RCCSMaterial, RCCSSThickness, RCCSMaxSize, RCCSRate, RCCSPerformance, RCCSCost $\}$.

Definition 4: Status attribute of the rectangular cutting cloud services, RCCSStatusInfo $=$ \{RCCSStatus, RCCSLoad, RCCSTaskInfo\}.

Definition 5: Service quality attributes of the rectangular cutting cloud services, RCCSQoS $=$ \{RCCSTimes, CSD, OTDR, FailureRate\}.

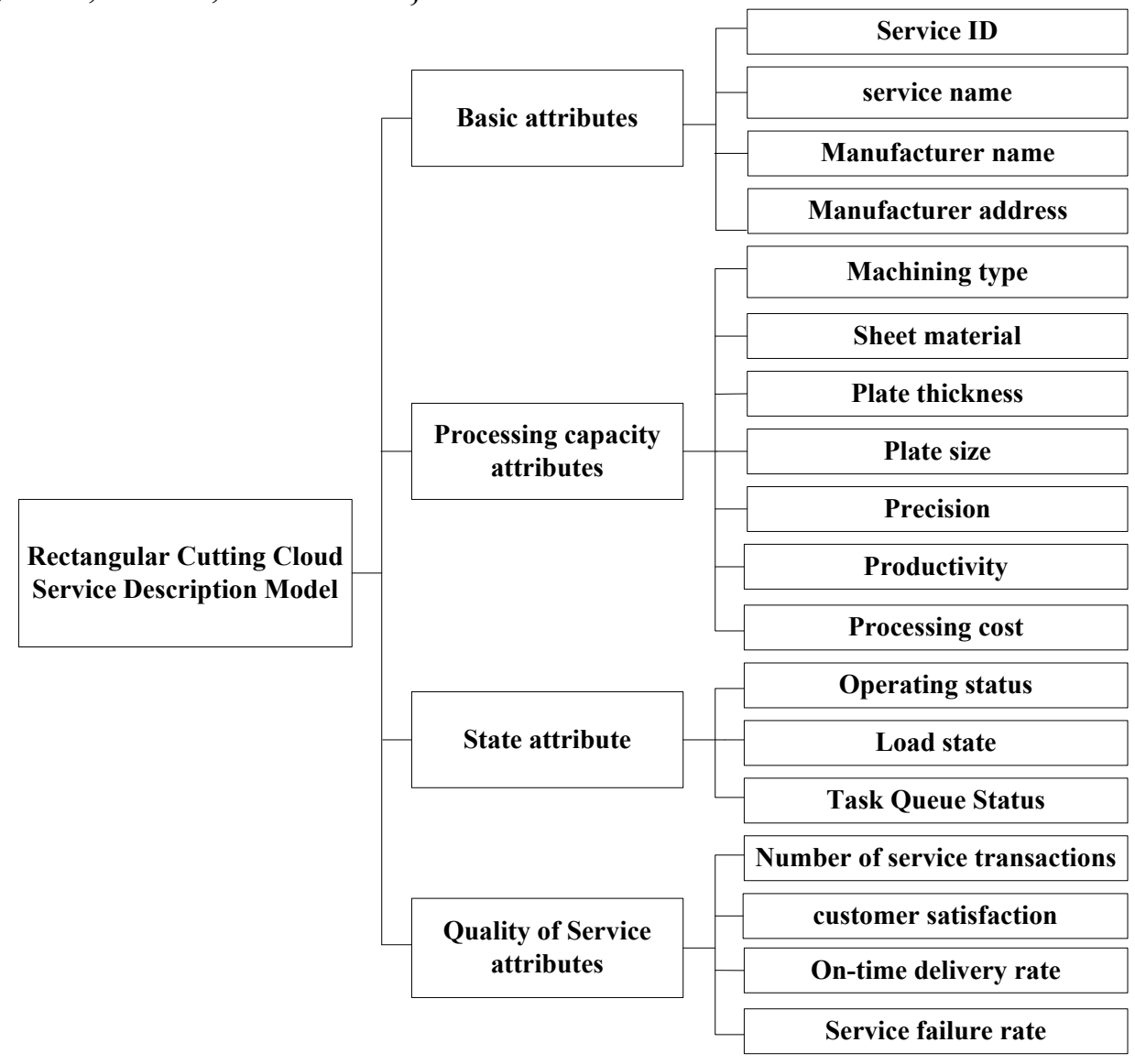

Figure 1 Rectangular cutting cloud service description model.

\section{Realization Framework of Rectangular Cutting Cloud Service Modeling}

According to the content and requirement analysis of cloud service modeling, the implementation framework based on three-layer structure model is used to represent the rectangular cutting cloud service modeling method, as shown in Figure 2.

1) Cloud service resource layer

Cloud service resource layer is the basis of rectangular cutting cloud service modeling, which is used to provide basic information data of cloud services. According to the formal definition method of the rectangular cutting cloud service description model, the information is extracted from the basic manufacturing resources (sheet material and processing equipment, etc.) to form the basic information, capability information, status information and service quality information of manufacturing resources.

2) Cloud services description layer

Cloud service description layer is the core and key of rectangle cutting cloud service modeling. This layer uses the STEP standard and system to describe the cloud service. The STEP standard provides a neutral mechanism suitable for data exchange, and is an international standard for product structure data representation and sharing. The semantic description and expression are expressed in the form of EXPRESS modeling language, which is driven by the modeling engine through the techniques of structured language definition, data structure description rules, data sharing and publishing mechanism and information transmission interaction standard[3]. 


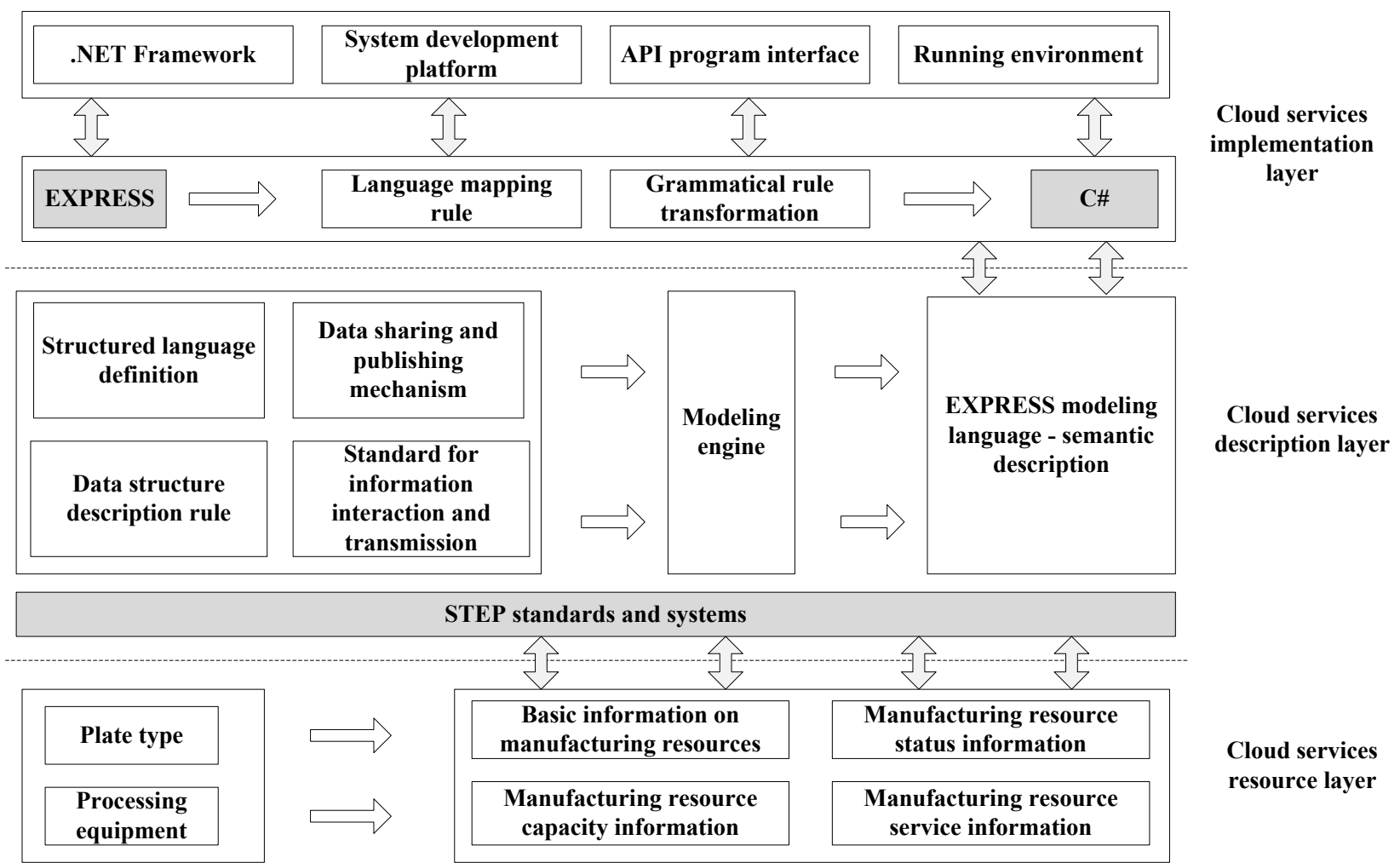

Figure 2 Realization framework of rectangular part cutting cloud service modeling.

The semantic description of cloud services using EXPRESS modeling language mainly includes description of type, model, entity, function, process. The following is a sample code for some semantic description of the rectangular cutting cloud service.

ENTITY Resource-basic-info;

Service-index: Text;

Service-name: Text;

Company-name: Text;

Company-address: Text;

END ENTITY;

ENTITY Resource-function-info;

Cutting-type: Text;

Plate-material: Text;

Plate-thickness: Text;

Plate-maxsize: Text;

\section{END ENTITY;}

3) Cloud service implementation layer

EXPRESS modeling language is very powerful in semantic description, but it cannot be used directly in the realization of terminal function modules, so it is necessary to code the EXPRESS modeling language. $\mathrm{C \#}$ is a programming language in the field of software design and system development is widely used. It has the advantages of easy to use and powerful, especially in the development of desktop applications, embedded software, network system etc. Therefore, based on the specific language mapping rules and syntax transformation rules, the EXPRESS is converted to the corresponding $\mathrm{C} \#$ code.

According to the nature of object oriented language, the mapping rules are set up in terms of class and object definition, definition of variable type, description of inheritance attribute, and the example code is as follows:

ENTITY Service;

a: REAL; 


\section{b: STRING; \\ c: REAL; \\ d: INTEGER; \\ END ENTITY;}

According to the mapping rules, convert to $\mathrm{C}$ \# code as follows:

Public Class Service \{

private double a;

private string $b$;

private double c;

private int d; \}

\section{The Rectangular Cutting Cloud Service Composition Process}

The rectangular cutting cloud service composition is based on the actual characteristics of the cutting task to select and match the existing services on the cloud manufacturing service platform. Its combination process is similar to the service composition process in the traditional network manufacturing mode, and usually including three stages: task decomposition, cloud service optimization, cloud service composition [4-6]. The combination process and architecture are shown in figure 3.
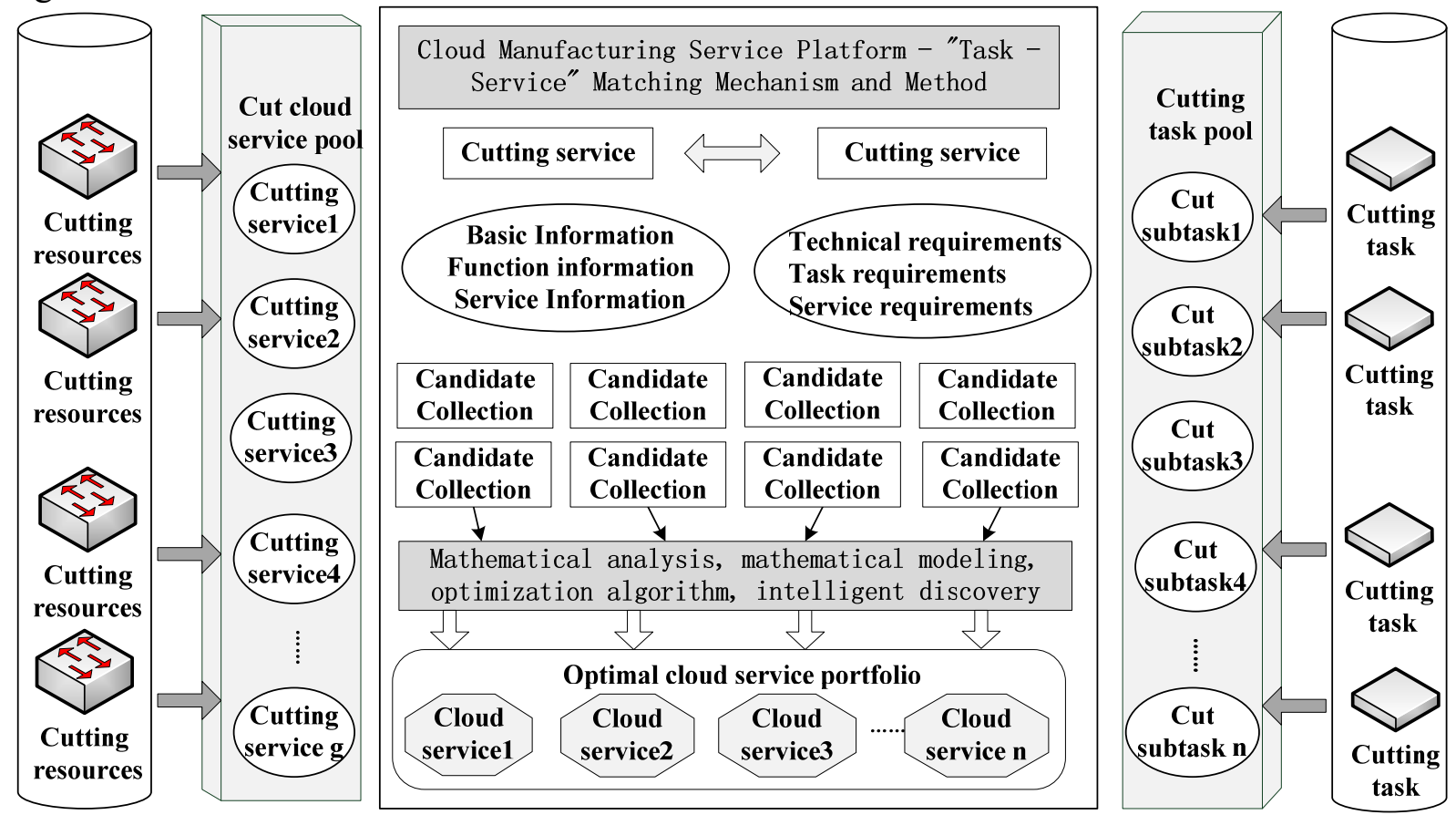

Figure 3 Process and architecture of rectangular cutting cloud service composition.

(1) Task decomposition

The workpiece segmentation task submitted by the client is decomposed into fine grained sub tasks that cannot be subdivided. Each subtask can be executed and completed by a single cloud service. The cutting task submitted by a client is named Task, then Task $=\left\{\right.$ Task $_{1}$, Task $_{2}, \ldots \ldots$, $\left.\operatorname{Task}_{n}\right\}, \mathrm{n}$ is the subtask number.

(2) Cloud service optimization

According to the matching rules of "cutting subtask - cloud manufacturing service", the cloud manufacturing service which does not satisfy the matching relation or the lower matching fitness value is filtered out to generate the cloud manufacturing service candidate collection for each cutting subtask. For the cutting subtask Task, the candidate set of cloud services satisfying its functional requirements can be represented as $S T S_{i}=\left\{S T S_{i 1}, S T S_{i 2}, \ldots \ldots, S T S_{i k}\right\}, \mathrm{k}$ is the number of candidate cloud services.

(3) Cloud service portfolio 
According to the method of mathematical analysis and mathematical modeling, the mathematical model of cloud service composition optimization is established, and the optimization algorithm is used to select the best cloud service from all the candidate cloud service sets to obtain an optimized cloud manufacturing service composition implementation scheme.

In the cloud service selection stage, this paper adopts the semantic web service matching method, and the matching of semantic service is divided into four levels: exact matching, insertion, including matching and non-matching.

Based on the above matching rules, the ability of cutting tasks mainly includes processing type, sheet material, plate thickness, sheet size, machining accuracy and so on. In the matching process, the matching accuracy of the cloud service and the cutting task needs to reach the "Exact" exact match level, if not satisfied, the cloud service is discarded.

\section{Conclusion}

This paper presents the modeling and composition process of cloud cutting rectangle service for cloud manufacturing, which solves the problem of description model of rectangular cutting cloud service and the realization framework of cloud service modeling. It is an important factor in the optimization of cloud file composition. The method proposed in this paper also has some reference for solving other similar problems.

\section{Acknowledgements}

This work was supported by the National Nature Science Foundation of China (Grant No. 51405133), the Province Science and Technology Research Projects of Henan (Grant No. 172102210213), and the Fundamental Research Funds for the Henan Provincial Colleges and Universities in Henan University of Technology (Grant No. 2016RCJH02).

\section{References}

[1] Jing, S.K., Jiang, H. and Xu, W.T. (2014) Consider Implementing the Reliability of Cloud Manufacturing Services Combining Algorithm. Journal of CAD \& CG, 26, 392-400.

[2] Yang, T. and Zhang, Y.F. (2015) Active Discovery and Agile Configuration Method for Manufacturing Services in Cloud Manufacturing. Computer Integrated Manufacturing System, 21, 1124-1133.

[3] Wang, X., Yin, C. and Li, X.B. (2016) Research on Modeling Method of Cloud Manufacturing Service for Machine Tool Equipments. Modern Manufacturing Engineering, 51-58.

[4] Ma, W.L., Wang, Z. and Zhao, Y.W. (2016) Cloud Manufacturing Service Composition Optimization Based on Improved Ant Colony Algorithm. Computer Integrated Manufacturing System, 22, 113-121.

[5] Su, K.K., Xu, W.S. and Li, J.Y. (2015) Optimal Allocation of Resources Based on Bi-level Programming in Manufacturing Environment. Computer Integrated Manufacturing System, 21, 1941-1952.

[6] Wu, Z.Y., Liu, X.X. and Li, L. (2012) Multi-objective Optimization for Task Assignment in Product Development. Control and Decision, 27, 598-602. 